\title{
Reactivity of the mesenteric bed arteries of normotensive rats exposed to chronic social stress
}

\author{
Angelika Puzserova ${ }^{1}$, Jozef Torok ${ }^{1}$, Ruzena Sotnikova ${ }^{2}$, Anna Zemancikova ${ }^{1}$ and Iveta Bernatova ${ }^{1}$ \\ ${ }^{1}$ Institute of Normal and Pathological Physiology, Centre of Excellence for Examination of Regulatory Role of Nitric Oxide \\ in Civilization Diseases, Slovak Academy of Sciences, Sienkiewiczova 1, 81371 Bratislava, Slovak Republic \\ ${ }^{2}$ Institute of Experimental Pharmacology and Toxicology, Slovak Academy of Sciences, Dubravska cesta 9, 84104 Bratislava, \\ Slovak Republic
}

\begin{abstract}
The aim of this study was to investigate the effects of chronic social stress on endotheliumdependent relaxation in the superior mesenteric artery (SMA) and its first branches (1MA) as well as on neurogenic contractions of SMA in adult, male Wistar-Kyoto (WKY) rats. Mesenteric arteries were isolated from control (living space: $480 \mathrm{~cm}^{2} /$ rat) or stressed rats exposed to 8-week-lasting crowding stress (living space: $200 \mathrm{~cm}^{2} / \mathrm{rat}$ ). Blood pressure (BP) and heart rate, determined by tail-cuff plethysmography, were not affected by crowding. Stress increased neurogenic contractions of SMA elicited by electrical stimulation of perivascular nerves and significantly elevated vasoconstriction induced by exogenous noradrenaline in SMA, without modulation of its endothelial function. In 1MA, nitric oxide (NO)-dependent component of endothelium-dependent relaxation to acetylcholine was investigated. In 1MA, stress failed to affect noradrenaline- and phenylephrine-induced vasoconstriction, total acetylcholine-induced relaxation as well as its NO-dependent and NO-independent components. Moreover, endothelium-independent sodium nitroprusside-induced relaxations of 1MA from the stressed rats did not differ from those of controls. In conclusion, chronic stress produced by crowding failed to induce an increase of BP, presumably because endothelial function of SMA and vascular function of small mesenteric arteries, which are rather important in BP regulation, remained preserved.
\end{abstract}

Key words: Crowding stress - Endothelium — Nitric oxide - Vasoactivity

\begin{abstract}
Abbreviations: AI, atherogenic index; AsA, ascorbic acid; HDL, high-density lipoproteins; LDL, low-density lipoproteins; $\mathrm{L}-\mathrm{NAME}, \mathrm{N}^{\mathrm{G}}$-nitro-L-arginine methyl ester; LVW, left heart ventricle; $1 \mathrm{MA}$, first branches of the superior mesenteric artery; $\mathrm{NO}$, nitric oxide; NOS, nitric oxide synthase; $\mathrm{PGI}_{2}$, prostacyclin; PSS, physiological salt solution; SHR, spontaneously hypertensive rats; SMA, superior mesenteric artery; TC, total cholesterol; TGL, triglycerides; VLDL, very-low-density lipoproteins; WKY, Wistar-Kyoto rats.
\end{abstract}

\section{Introduction}

Although the mechanisms involved in stress-induced cardiovascular disorders, including hypertension, are not well defined, there are studies showing a possible involvement of peripheral vascular changes. Since the endothelium plays an

Correspondence to: Angelika Puzserova, Institute of Normal and Pathological Physiology, Centre of Excellence for Examination of Regulatory Role of Nitric Oxide in Civilization Diseases, Slovak Academy of Sciences, Sienkiewiczova 1, 81371 Bratislava, Slovak Republic

E-mail: angelika.puzserova@savba.sk important role in the regulation of vascular tone via synthesis and release of vasoactive substances, endothelial dysfunction may contribute to the increased peripheral resistance in stress-induced hypertension. Moreover, endothelial dysfunction appears to play a pathogenic role in the initial development of atherosclerosis and a number of studies have shown that nitric oxide (NO)-dependent vasodilator function is impaired in patients with risk factors for atherosclerosis (Caramori and Zago 2000). It was also shown that chronic stress, in addition to hypertension, may induce proatherogenic changes (Neves et al. 2009). There are studies showing that psychosocial factors may influence atherogenesis even in the absence of elevated serum lipids (Kaplan et al. 1983). The 
finding that chronic mild stress is associated with increased plasma levels of total cholesterol and NO deficiency (Neves et al. 2009) suggests that hypercholesterolemia may be a link between stress and endothelial dysfunction.

NO, among other factors, seems to be involved in stress physiology, and alterations in NO availability apparently take part in stress-related disease processes (Leza et al. 1998; Esch et al. 2002). NO is known to counterbalance the effect of sympathetic stimulation at the peripheral (Török 2008) as well as central level (Gerová et al. 1995; Esch et al. 2002). NO plays also an important role in hypothalamic-pituitary-adrenal axis stimulation under basal and stress conditions (Bugajski et al. 2006; Gadek-Michalska and Bugajski 2008; GadekMichalska et al. 2008). The evidence that NO production may be considerably modified in stress and during adaptation to diverse stressors has entailed the hypothesis that NO plays an important role in adaptive responses of the organism as a stress-limiting molecule (Malyshev and Manukhina 1998). Activation of the local L-arginine/NO system may produce antagonistic interactions of NO with different vasoconstrictors, such as catecholamines (Leza et al. 1998; Chies et al. 2003), and could thus counterbalance the effect of stress on blood pressure (Scheuer 2010). Recently we also provided evidence that chronic crowding improved endothelium-dependent acetylcholine-induced vasorelaxation of the first branches of the superior mesenteric arteries (1MA) of Wistar-Kyoto rats (Bernátová et al. 2007b). However, at least three different vasodilating factors are known to be released by the endothelium after exposure to acetylcholine, i.e. NO, prostacyclin $\left(\mathrm{PGI}_{2}\right)$, and endothelium-derived hyperpolarizing factor(s) (Mitchell et al. 2008; Török 2008). From our previous experiments it is yet not clear which of these vasodilating factors were increased in 1MA. On the other hand, elevated NO production need not always be associated with better $\mathrm{NO}$ bioavailability due to increased concentration of reactive oxygen species (ROS). An elevated level of ROS can inactivate NO and thus contribute to endothelial dysfunction (Török 2008; Bernatova et al. 2009). In our previous experiments, stress-related vascular reactivity and endothelial function were measured in the presence of the well known antioxidant ascorbic acid (AsA) in working solution (Bernátová et al. 2007b) to prevent oxidation of catecholamines (Bernatova et al. 2009). However, AsA might artificially reverse endothelial dysfunction by improvement of the oxidative status, resulting in better NO bioavailability (Bernatova et al. 2009). Therefore in this study, we investigated vascular reactivity of $1 \mathrm{MA}$ without adding AsA to working solution.

Crowding is a relatively mild stressor, but it effectively affects the hypothalamic-pituitary-adrenal axis and sympathoadrenal system, known to be the main stress systems in both humans and experimental animals (Bugajski 1999; Djordjević et al. 2003; Dronjak et al. 2004). As to blood pressure, crowding increased it in rats with a positive family history of hypertension (Bernatova et al. 2007a) as well as in humans (D’Atri et al. 1981).

This study was aimed at investigating the effect of chronic social stress on the mesenteric arteries and its role in the initiation of stress-related hypertension, considering also changes in cholesterol metabolism. As the mesenteric circulation is regulated by several factors including the endothelium and sympathetic nervous system, vascular responses to endothelium-dependent and endothelium-independent vasodilators (acetylcholine and sodium nitroprusside, respectively) were determined. Additionally, vasoconstrictor function was investigated using noradrenaline and the selective $\alpha_{1}$-adrenoceptor agonist phenylephrine, as well as electrical stimulation of perivascular nerves to simulate neurogenic contractions.

\section{Materials and Methods}

\section{Ethical approval and animals}

All procedures were performed in accordance with the guidelines approved by the State Veterinary and Food Administration of the Slovak Republic and they were in compliance with the National Institutes of Health Guide for Care and Use of Laboratory Animals.

All rats used in the present study were born in a certified animal facility (Institute of Normal and Pathological Physiology) in order to maintain the same environmental background. The rats were housed in an air-conditioned room at constant temperature $\left(22 \pm 2^{\circ} \mathrm{C}\right)$ and humidity $(45-60 \%)$ on a 12:12 h light/dark cycle with lights on from 6.00 to $18.00 \mathrm{~h}$ and maintained on a standard laboratory rat chow and tap water ad libitum.

\section{Experimental design and stress model}

Eighteen rats were used in the experiment. After weaning $\left(25^{\text {th }}\right.$ day), male Wistar-Kyoto (WKY) rats were kept in groups of four per cage $\left(35 / 55 / 20 \mathrm{~cm}, 480 \mathrm{~cm}^{2} /\right.$ rat $)$ under standard housing conditions. At the beginning of the experiment, 12-week-old rats were randomly divided into two groups, a control and a stressed group. Controls were kept in groups of four rats per cage $(35 / 55 / 20 \mathrm{~cm})$. Rats exposed to crowding were kept in conditions of reduced living space from $480 \mathrm{~cm}^{2} /$ rat (controls) to $200 \mathrm{~cm}^{2} /$ rat (Bernatova et al. 2007a, 2010) for eight weeks. During the experiment, the rats were submitted to procedures related to routine animal care and blood pressure measurements.

After 8 weeks of experiment, the rats were killed by decapitation after a brief $\mathrm{CO}_{2}$ anaesthesia, between 7.30-9.30 h. Wet weights of the left heart ventricle (LVW) were determined for calculation of their relative weights (LVW/tibial length) to evaluate the degree of cardiac hypertrophy. 
Blood pressure and heart rate

Systolic blood pressure and heart rate were determined noninvasively in conscious rats by the tail-cuff method (using the Statham Pressure Transducer P23XL, Hugo Sachs, Germany) before experiment (basal) and after the $1^{\text {st }}, 3^{\text {rd }}, 6^{\text {th }}$ and $8^{\text {th }}$ week of experiment. One week before experimentation, the rats were handled and accustomed to the tail-cuff procedure of blood pressure recording in three independent sessions to avoid non-specific stress. Blood pressure and heart rate were measured between $9.00-12.00 \mathrm{~h}$ and calculated as the average value of 5-6 measurements.

\section{Cholesterol content analysis}

Samples of the liver and skeletal muscle from the hind limb were collected and kept at $-80^{\circ} \mathrm{C}$ until analysis. Then tissues were homogenised in a chloroform-methanol mixture (1:1) and total cholesterol was determined according to Ulicná et al. (2003).

\section{Analytic methods and atherogenic index}

After decapitation, blood was collected in heparin-coated tubes. The plasma was frozen and stored at $-20^{\circ} \mathrm{C}$ until determination of plasma lipid concentrations by a standard automated technique using Biocode-Hycel Analyzer Model AL Plus. Plasma triglycerides (TGL), total cholesterol (TC) and high-density lipoproteins (HDL) were determined by the commercially available kits. All kits were purchased from Spinreact Company (Spain). Concentration of very-low-density lipoproteins (VLDL) was calculated from TGL by Friedewald's formula: $\mathrm{VLDL}=\mathrm{TGL} / 5$. Plasma low-density lipoproteins (LDL) were calculated by Friedewald's formula: $\mathrm{LDL}=\mathrm{TC}$ - HDL - (TGL/2) (Friedewald et al. 1972; Kamgang et al. 2005). The atherogenic index (AI) was determined using the formula: $\mathrm{AI}=\mathrm{TC}-\mathrm{HDL} / \mathrm{TC}$ according to Neves et al. (2009).

\section{In vitro assessment of vascular reactivity by wire myograph}

The first branches of the superior mesenteric arteries (1MA) were carefully dissected out, immediately immersed in modified cold $\left(4^{\circ} \mathrm{C}\right)$ physiological salt solution (PSS) and cleaned of connective tissue. The arteries were then cut into segments $(1.54 \pm 0.07 \mathrm{~mm}$ long) and mounted as ring-shaped preparations in Mulvany-Halpern's small vessel wire myograph (Mulvany and Halpern 1977) chamber (Dual Wire Myograph System 410A, DMT A/S, Aarhus, Denmark) to determine the vascular reactivity under isometric conditions. The relation between resting wall tension and internal circumference and subsequently the effective internal lumen diameter were determined by a computer-assisted normalisation procedure to set the pretension of the arteries after 30-min equilibration in oxygenated $\left(95 \% \mathrm{O}_{2}, 5 \% \mathrm{CO}_{2}\right.$ mixture) PSS, composition in $\mathrm{mmol} / \mathrm{l}: \mathrm{NaCl}$
118.99, $\mathrm{KCl} 4.69, \mathrm{NaHCO}_{3} 25, \mathrm{MgSO}_{4} \cdot 7 \mathrm{H}_{2} \mathrm{O} 1.17, \mathrm{KH}_{2} \mathrm{PO}_{4}$ 1.18, $\mathrm{CaCl}_{2} \cdot 2 \mathrm{H}_{2} \mathrm{O} 2.5, \mathrm{Na}_{2}$ EDTA 0.03, glucose 5.5, pH 7.4 at $37^{\circ} \mathrm{C}$. The changes in wall tension (active wall tension) were calculated as measured force divided by the double segment length and expressed in $\mathrm{mN} / \mathrm{mm}$. Resting wall tension, which arises from the properties of the passive elements in the vascular wall, was also determined after the normalisation procedure (Mulvany and Halpern 1977).

\section{Experimental protocol for functional studies of the first branches of the superior mesenteric artery}

Before starting experimentation, the vessels were allowed to stabilise in PSS for 30 minutes. The experimental protocol consisted of the following steps: 45 min after normalisation, PSS was changed to high-potassium PSS ( $125 \mathrm{mmol} / \mathrm{l}$, for $2 \mathrm{~min}$ ) in which $\mathrm{NaCl}$ in normal PSS was exchanged for an equimolar concentration of $\mathrm{KCl}$, followed by wash-out with PSS (15 min). After noradrenaline addition $(10 \mu \mathrm{mol} / \mathrm{l}$, waiting to plateau) and wash-out (PSS, $20 \mathrm{~min}$ ), pre-constriction was induced by phenylephrine $(3 \mu \mathrm{mol} / \mathrm{l})$. When the contraction of $1 \mathrm{MA}$ to phenylephrine reached a steady state, increasing concentrations of the vasodilator acetylcholine $(0.001$ to $10 \mu \mathrm{mol} / \mathrm{l})$ were added in cumulative manner to perform endothelium-dependent concentration-response curves. When the concentration-relaxation curve was completed, the drugs were washed-out (PSS, $20 \mathrm{~min}$ ) and the same procedure was repeated after 25-min pre-incubation with the nitric oxide synthase (NOS) inhibitor $\mathrm{N}^{\mathrm{G}}$-nitro-L-arginine methyl ester (L-NAME, $300 \mu \mathrm{mol} / \mathrm{l}$ ) in the bath medium. After the last acetylcholine concentration, the NO donor sodium nitroprusside $(100 \mu \mathrm{mol} / \mathrm{l})$ was added to the bath to check endothelium-independent NO vasodilator function. The NO-dependent component of endothelium-dependent relaxation was calculated as the difference between acetylcholine-induced relaxation before and after acute L-NAME pre-treatment and expressed as the area under the curve (AUC, arbitrary units) based on individual concentration-response curves (Puzserova and Bernatova 2010). In our preliminary experiments we ruled out tachyphylaxis (Hansen and Nedergaard 1999) in two consecutive concentration-response curves for acetylcholine in phenylephrine pre-contracted 1MA (data not shown). The extent of vasorelaxation was expressed as the percentage of phenylephrine-induced contraction. Vasoconstrictions were determined as maximal tension and were expressed as active wall tension in $\mathrm{mN} / \mathrm{mm}$ (Mulvany and Halpern 1977). Responses to each drug concentration were always allowed to stabilise before the addition of a subsequent dose of the same drug or another drug (Webb et al. 1987).

\section{In vitro assessment of vascular reactivity in organ chamber}

The superior mesenteric artery (SMA) was dissected out and immediately immersed in cold modified Krebs solution 
(containing in mmol/l: $\mathrm{NaCl} 118, \mathrm{KCl} 5, \mathrm{NaHCO}_{3} 25, \mathrm{MgSO}_{4}$ $1.2, \mathrm{KH}_{2} \mathrm{PO}_{4} 1.2, \mathrm{CaCl}_{2} 2.5, \mathrm{CaNa}_{2}$ EDTA 0.03 , glucose 11 and AsA 0.55) for isometric tension studies. Connective tissue was carefully removed from the SMA and it was cut into 3-mmlong ring segments with intact endothelium. For measurement of isometric tension, the rings were suspended between two stainless-steel hooks in an organ chamber $(20 \mathrm{ml})$ filled with oxygenated $\left(95 \% \mathrm{O}_{2}\right.$ and $5 \% \mathrm{CO}_{2}$ mixture) modified Krebs solution maintained at $37^{\circ} \mathrm{C}$. Isometric tension was monitored continuously using a high-sensitivity force-displacement transducer Sanborn FT 10. The arterial rings were initially equilibrated under resting tension of $10 \mathrm{mN}$ for $60-90 \mathrm{~min}$, and the buffer was changed every 15 min (Čačányiová et al. 2011).

\section{Experimental protocol for contraction reactivity of the SMA}

After the equilibration period, vasoconstrictor responses were elicited by exogenous noradrenaline applied cumulatively into the organ chamber solution or by endogenous noradrenaline released from perivascular sympathetic nerve terminals during transmural electric stimulation. For electric stimulation the trains of $0.5 \mathrm{~ms}$ rectangular pulses of supramaximal intensity $(>30 \mathrm{~V})$ at frequencies $1-32 \mathrm{~Hz}$ for a period of $20 \mathrm{~s}$ were used. After the experimental protocol, SMA rings were stimulated with high concentration of $\mathrm{K}^{+}$ $(100 \mathrm{mmol} / \mathrm{l})$ in depolarising solution $(\mathrm{KCl}$ replaced $\mathrm{NaCl}$ on equimolar basis in the Krebs solution). The maximal tension achieved with depolarising solution was set as $100 \%$ to express the active tension generated by endogenous noradrenaline (Siddegowda et al. 2007).

\section{Experimental protocol for relaxation reactivity of the SMA}

Acetylcholine-induced responses were examined in the other segments of the SMA in modified Krebs solution without AsA (Sotnikova et al. 2006). The concentration-response curve to acetylcholine was constructed without and with NOS and cyclooxygenase inhibitors (30 min incubation: 100 $\mu \mathrm{mol} / \mathrm{l} \mathrm{L}-\mathrm{NAME}$ and $10 \mu \mathrm{mol} / \mathrm{l}$ indomethacin) in phenylephrine $(1 \mu \mathrm{mol} / \mathrm{l})$-contracted SMA. After the last acetylcho- line concentration, sodium nitroprusside $(100 \mu \mathrm{mol} / \mathrm{l})$ was added to the bath to check endothelium-independent NO vasodilator function.

\section{Drugs}

All the chemicals used were purchased from Sigma-Aldrich (Germany) except noradrenaline hydrogenotartras (Zentiva, Czech Republic). Indomethacin was dissolved in 5\% $\mathrm{NaHCO}_{3}$. All other drugs were dissolved in distilled water and concentrations were expressed as final concentration in the myograph or organ chamber.

\section{Statistical analysis}

Data are presented as group mean values \pm standard error of the mean (SEM). Unpaired Student's $t$-test was used for comparison of means of two groups and two-way analysis of variance (ANOVA) for comparison of concentration (or frequency)-response curves. In case of significant results of two-way ANOVA, vertical contrast (pairwise comparisons) with Bonferroni adjustment were performed. Means were considered to differ significantly when $p<0.05$. The concentration-response curves were fit by non-linear regression using four parameter logistic equations with GraphPad Prism 5.0 software (San Diego, CA, USA). Changes in 1MA and SMA sensitivity to acetylcholine and noradrenaline were evaluated by determining the mean negative logarithm of molar concentration producing $50 \%$ of maximal response $\left(\mathrm{pD}_{2}\right)$ (Siddegowda et al. 2007; Neves et al. 2009). $\mathrm{pD}_{2}$ were determined using GraphPad Prism 5.0.

\section{Results}

Basic biometric, cardiovascular and biochemical parameters

Before experiment, the body weight of the control and crowded group of rats was $308 \pm 5 \mathrm{~g}$ and $318 \pm 5 \mathrm{~g}$, respectively. Crowding significantly reduced the body weight gain at the end of the experiment (Table 1). Basal blood pressure

Table 1. Effect of chronic social stress on basic biometric, cardiovascular and biochemical parameters of Wistar-Kyoto rats

\begin{tabular}{lcc}
\hline & Control & Stress \\
\hline Relative BW gain $(\%)$ & $32 \pm 1$ & $18 \pm 2^{\star}$ \\
LVW/tibia length $(\mathrm{mg} / \mathrm{mm})$ & $14.04 \pm 0.48$ & $13.24 \pm 0.35$ \\
CHOL in the liver $(\mathrm{mmol} / \mathrm{kg})$ & $21.58 \pm 0.62$ & $23.55 \pm 0.67$ \\
CHOL in the skeletal muscle $(\mathrm{mmol} / \mathrm{kg})$ & $5.51 \pm 0.22$ & $4.91 \pm 0.28$ \\
Atherogenic index & $1.04 \pm 0.07$ & $1.10 \pm 0.08$ \\
lMA normalised diameter $(\mu \mathrm{m})$ & $413.49 \pm 22.06$ & $361.22 \pm 17.63$ \\
1MA resting wall tension $(\mathrm{mN} / \mathrm{mm})$ & $1.12 \pm 0.24$ & $0.93 \pm 0.02$ \\
\hline
\end{tabular}

Values represent mean \pm SEM of 6-10 rats. BW, body weight; CHOL, cholesterol; LVW, left heart ventricle weight; 1MA, first branches of the superior mesenteric artery. ${ }^{*} p<0.05$, compared to respective value in control rats. Data were analysed by Student's $t$-test. 
A

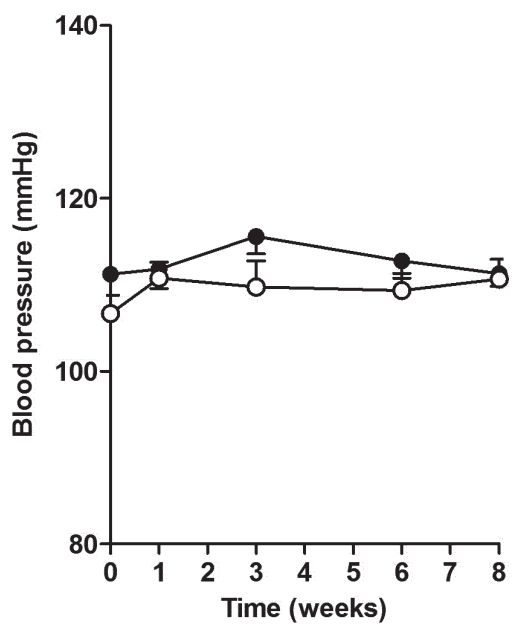

B

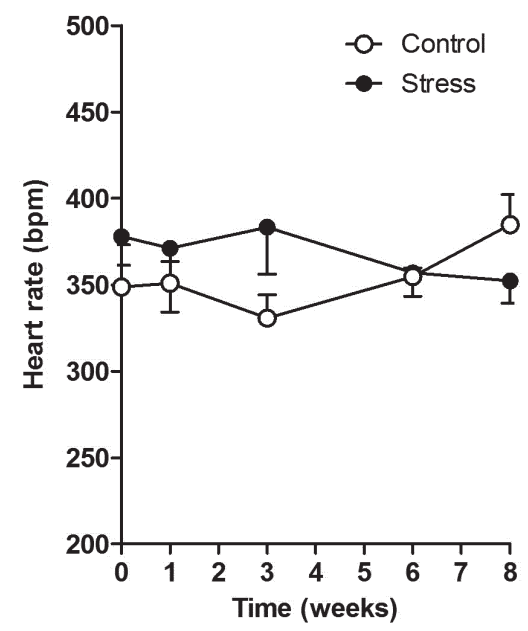

Figure 1. Effect of stress on blood pressure (A) and heart rate (B). Values represent mean \pm SEM of $8-10$ rats. Data were analysed by two-way ANOVA test, followed by Bonferroni post hoc test.

and heart rate of control and stressed rats before experiment were $107 \pm 4 \mathrm{mmHg}$ and $111 \pm 3 \mathrm{mmHg}$; and $349 \pm 24 \mathrm{bpm}$ and $378 \pm 17 \mathrm{bpm}$, respectively. Chronic crowding failed to alter blood pressure, heart rate (Fig. 1A,B), relative left ventricle weight (Table 1), as well as the normalised internal diameter at $100 \mathrm{mmHg}$ and resting wall tension of $1 \mathrm{MA}$ $v s$. the control group (Table 1). Similarly, no differences were observed in cholesterol content in the liver, skeletal muscle (Table 1) and blood plasma (Fig. 2). Plasma concentrations of TGL and VLDL were significantly decreased in the stressed group ( $p<0.05$, Fig. 2$)$ with no statistical difference in the plasma levels of TC, HDL and LDL after chronic stress. AI was also comparable in stressed and control rats (Table 1).

\section{Endothelium-dependent and-independent vasorelaxation of $1 M A$}

There were no differences in the maximal responses to acetylcholine, based on the individual concentration-response curves before or after pre-incubation with L-NAME between the control and stressed group (Table 2). Chronic crowding failed to affect the concentration-response curve to acetylcholine (statistical difference between groups was found only at one concentration: $0.01 \mu \mathrm{mol} / \mathrm{l}$ ) (Fig. 3A). However, stress decreased the $\mathrm{pD}_{2}$ value of acetylcholine compared to control (Fig. 3A). Since vasorelaxation, expressed as a percentage of pre-constriction, is inversely related to initial tension, we expressed relaxing responses also as absolute values ( $\mathrm{mN} / \mathrm{mm}$ ) (Webb et al. 1987; Hansen and Nedergaard 1999) to warrant that changes measured were endothelium-dependent and not related to

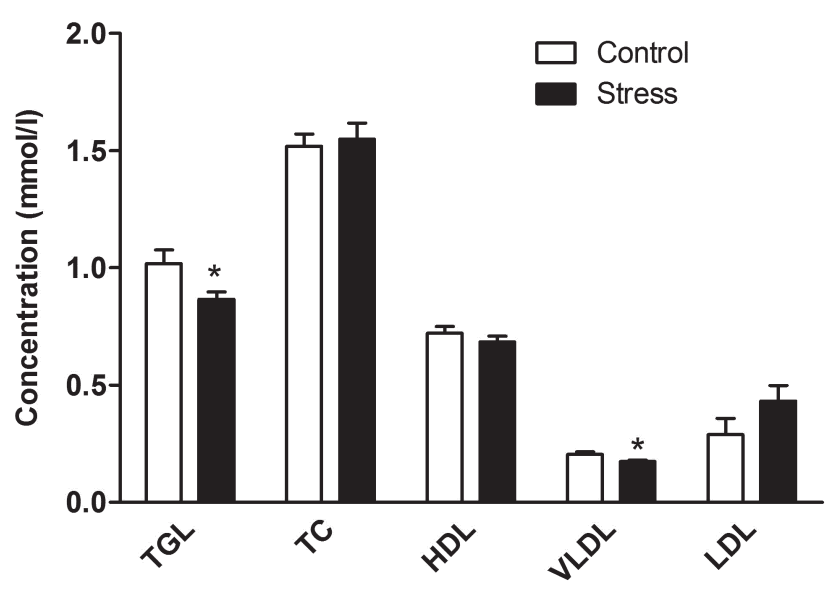

Figure 2. Effect of chronic social stress on plasma concentrations of triglycerides (TGL), total cholesterol (TC), high-density lipoprotein (HDL), very low-density lipoprotein (VLDL) and low-density lipoprotein (LDL). Values represent mean \pm SEM of 8-10 rats. Data were analysed by Student's $t$-test. ${ }^{\star} p<0.05$, compared to respective value in control rats.

the extent of pre-constriction. Interestingly, no functional changes of the endothelium in the 1MA were seen after crowding, when evaluated in absolute values (data not shown). Thus the results suggest that chronic crowding stress did not modify bioavailability of endothelial factors, although it decreased the $\mathrm{pD}_{2}$ value of acetylcholine-induced vasorelaxations when expressed in relative values. Blockade of NO synthesis by L-NAME reduced responses to low concentrations of acetylcholine (Fig. 3B). The NO- 
dependent and $\mathrm{NO}$-independent component of relaxation was similar in rings from stressed and control rats (Fig. 3C,D). Crowding stress had no significant effect on endothelium-independent sodium nitroprusside-induced relaxation of $1 \mathrm{MA}$ (Table 2 ).

A

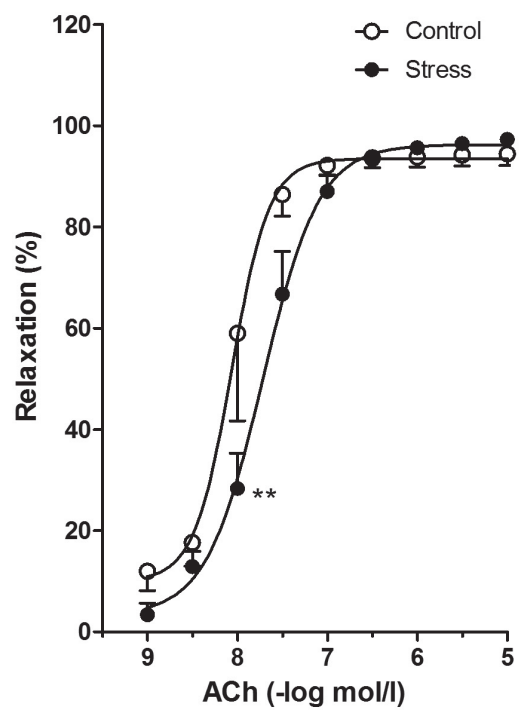

$\mathbf{C}$

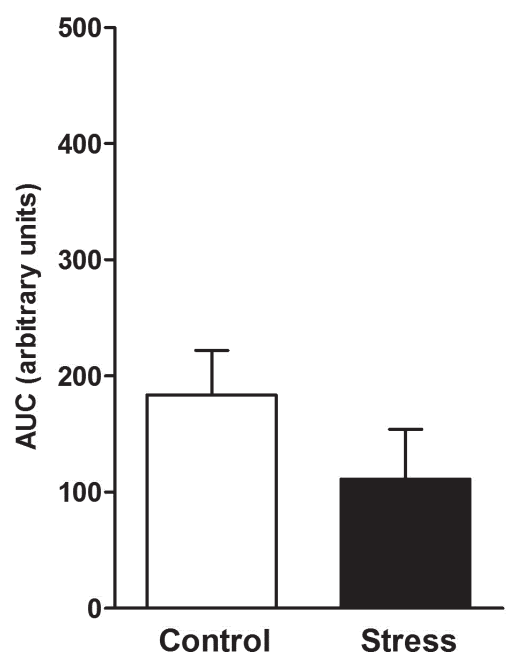

Vascular responsiveness to different vasoconstrictors of $1 \mathrm{MA}$ and SMA

Vascular reactivity (noradrenaline-induced vasoconstriction and phenylephrine-induced vasoconstriction) was investi-

B

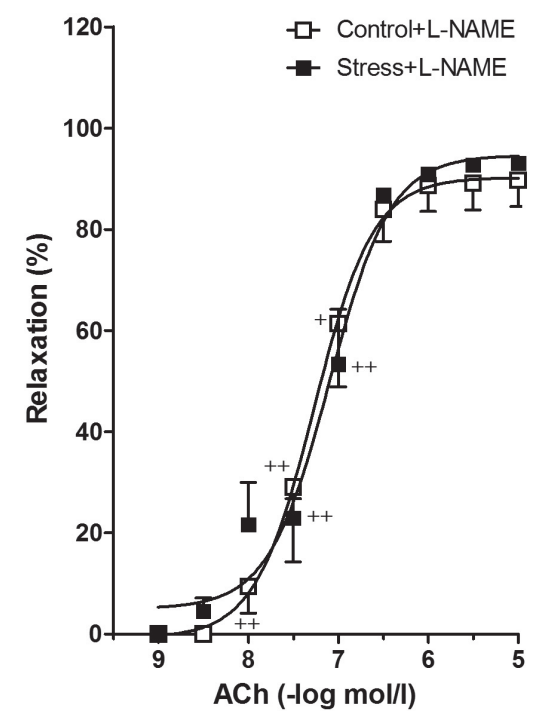

D

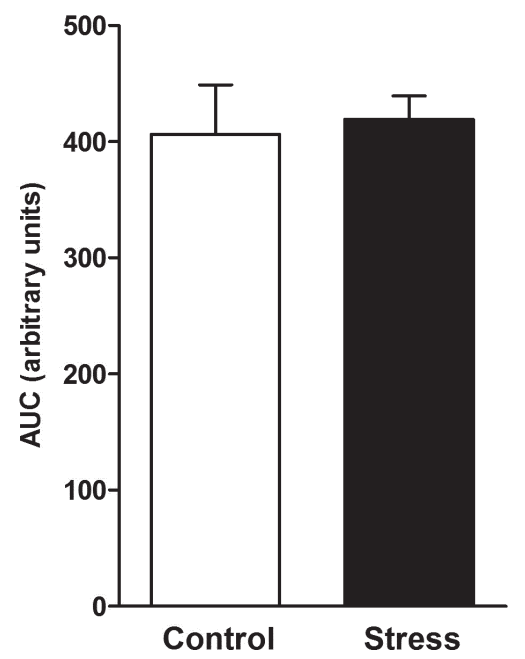

Figure 3. Effect of chronic social stress on acetylcholine (ACh)-induced endothelium-dependent relaxations in phenylephrine (PE, $3 \mu \mathrm{mol} / \mathrm{l}$ )-contracted first branches of the superior mesenteric artery segments before (A) and after (B) incubation with the nitric oxide synthase inhibitor $\mathrm{N}^{\mathrm{G}}$-nitro-L-arginine methyl ester (L-NAME), nitric oxide (NO)-dependent component of acetylcholine-induced relaxations (C) and NO-independent component of acetylcholine-induced relaxations (D). Mean values of the $\mathrm{pD}_{2}$ of $\mathrm{ACh}$ in the absence of L-NAME were $8.06 \pm 0.02$ (Control), $7.72 \pm 0.03$ (Stress, $p<0.01 v s$. Control) and in the presence of L-NAME were $7.27 \pm 0.02$ (Control $+\mathrm{L}-\mathrm{NAME}, p<$ 0.01 vs. Control) and $7.10 \pm 0.10$ (Stress+L-NAME, $p<0.01$ vs. Stress). Values represent mean \pm SEM of $6-7$ rats. Concentration-response curves were analysed by two-way ANOVA, followed by Bonferroni post hoc test. AUC was analysed by Student's $t$-test. ${ }^{* *} p<0.01$, compared to respective value in control rats; ${ }^{+} p<0.05,{ }^{++} p<0.01$, compared to respective value in control or stressed rats without L-NAME. AUC, area under the curve; $\mathrm{pD}_{2}$, negative logarithm of the molar concentration of agonist producing 50 percent of maximal response. 
Table 2. Effect of chronic social stress on vascular constrictions and maximal vascular relaxations of the first branches of the superior mesenteric artery (1MA) of Wistar-Kyoto rats

\begin{tabular}{llcc}
\hline & & Control & \multicolumn{1}{c}{ Stress } \\
\hline & & \multicolumn{2}{c}{$(\mathrm{mN} / \mathrm{mm})$} \\
Vasoconstrictors & Noradrenaline & $4.86 \pm 0.53$ & $4.78 \pm 0.32$ \\
& Phenylephrine before L-NAME & $3.86 \pm 0.62$ & $4.28 \pm 0.24$ \\
& Phenylephrine after L-NAME & $4.45 \pm 0.48$ & $4.40 \pm 0.16$ \\
\cline { 2 - 4 } Vasodilators & Maximal ACh before L-NAME & & $(\%)$ \\
& Maximal ACh after L-NAME & $94.87 \pm 2.12$ & $97.29 \pm 0.52$ \\
& SNP & $89.70 \pm 5.23$ & $93.08 \pm 0.39$ \\
\hline
\end{tabular}

Vascular constrictions induced by noradrenaline $(10 \mu \mathrm{mol} / \mathrm{l})$ and phenylephrine $(3 \mu \mathrm{mol} / \mathrm{l})$. Maximal vascular relaxations induced by acetylcholine (ACh) and sodium nitroprusside (SNP, $100 \mu \mathrm{mol} / \mathrm{l}$ ) of phenylephrine-contracted 1MA of Wistar-Kyoto rats. Values represent mean \pm SEM of 6-7 rats. L-NAME, $\mathrm{N}^{\mathrm{G}}$-nitro-L-arginine methyl ester $(300 \mu \mathrm{mol} / \mathrm{l}, 25 \mathrm{~min})$. Data were analysed by Student's $t$-test.

gated in 1MA. Crowding had no effect on noradrenalineand phenylephrine-induced (with or without L-NAME) vasoconstriction in $1 \mathrm{MA}$ (Table 2). No significant differences in phenylephrine-induced vasoconstriction were observed after L-NAME pre-incubation.

Noradrenaline-induced vasoconstriction, however, revealed significant differences in the SMA. Stress significantly elevated vasoconstriction induced by application of cumulative concentrations of exogenous noradrenaline (Fig. 4A).
Stress induced increase in maximal contractions as well as in $\mathrm{pD}_{2}$ values compared to control, indicating significant changes in sensitivity to noradrenaline (Fig. 4A).

\section{Neurogenic contractions of the SMA}

SMA rings responded to electrical stimulation of perivascular sympathetic nerve terminals by frequency-dependent contractions. The influence of crowding stress on neurogenic contrac-
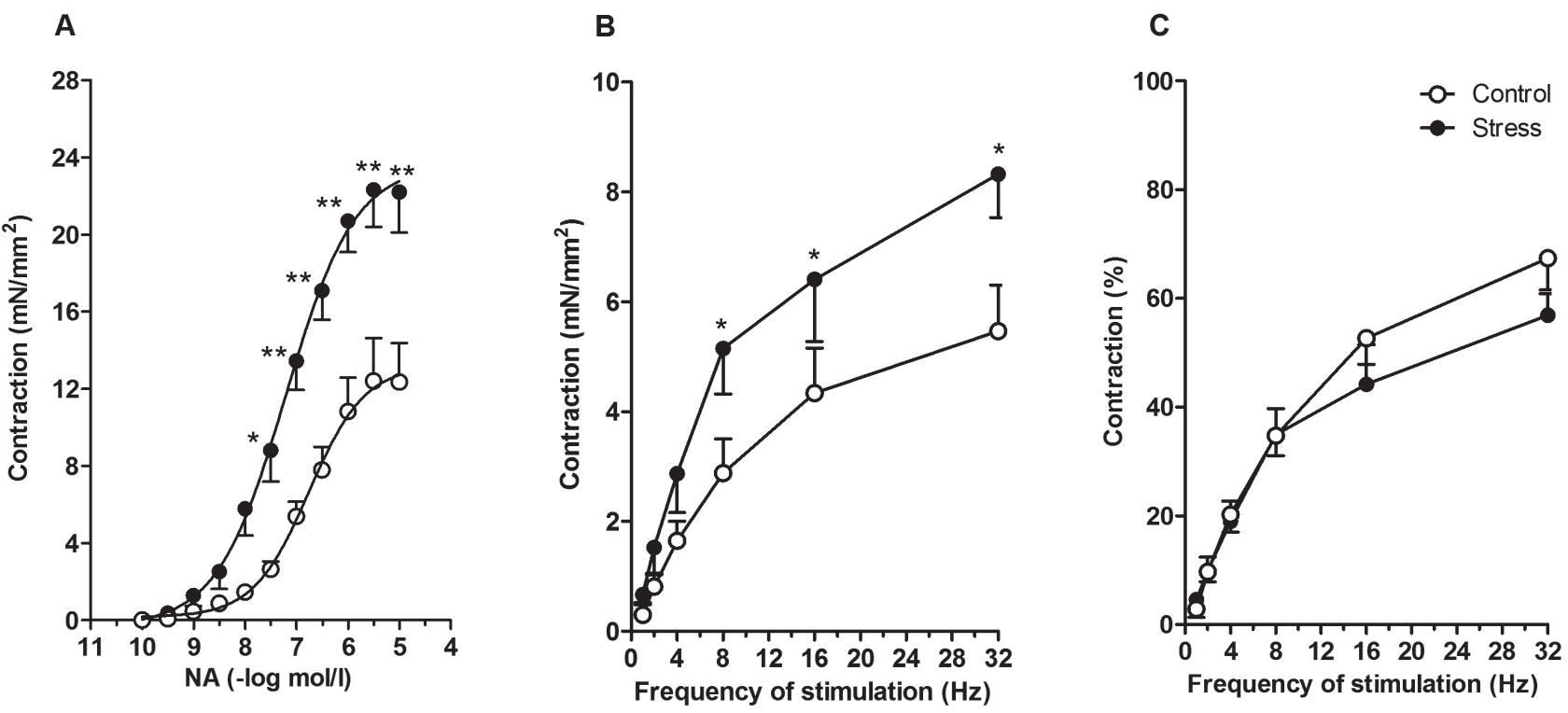

Figure 4. Contractions to exogenous noradrenaline and neurogenic contractions induced by endogenous noradrenaline of the isolated superior mesenteric artery submitted to chronic social stress. Concentration-response curve of noradrenaline-induced vasoconstriction, calculated as absolute tension (A). Mean values of the $\mathrm{pD}_{2}$ of NA were $6.75 \pm 0.05$ (Control) and $7.21 \pm 0.05$ (Stress, $p<0.01 v s$. Control). Frequency-response curves were constructed for absolute contractions $(\mathbf{B})$ and relative contractions calculated to maximal $\mathrm{KCl}(100$ $\mathrm{mmol} / \mathrm{l}$ ) contractions $(\mathbf{C})$. Values represent mean \pm SEM of 5-6 rats. Data were analysed by two-way ANOVA test, followed by Bonferroni post hoc test. ${ }^{*} p<0.05,{ }^{* *} p<0.01$, compared to respective value in control rats. $\mathrm{pD}_{2}$, negative logarithm of the molar concentration of agonist producing 50 percent of maximal response. 
A

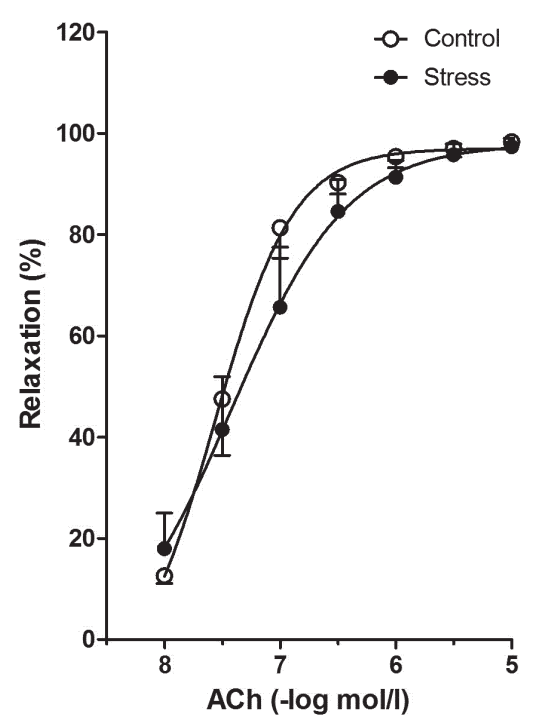

B

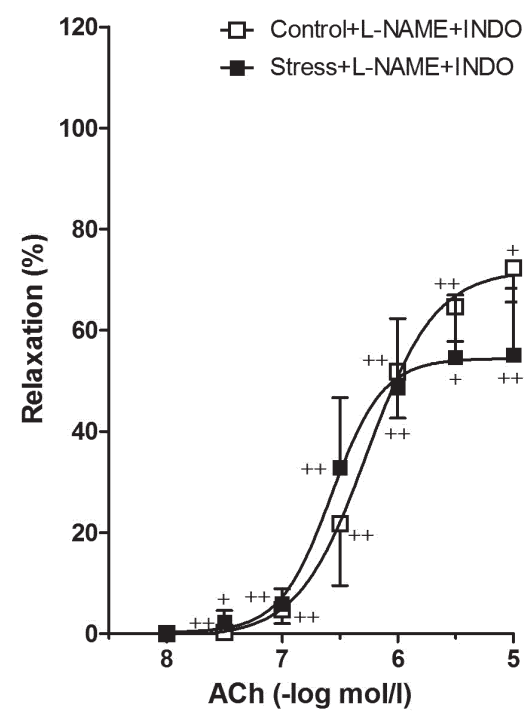

Figure 5. Effect of chronic social stress on acetylcholine (ACh)-induced endothelium-dependent relaxations in phenylephrine (PE, 1 $\mu \mathrm{mol} / \mathrm{l})$-contracted superior mesenteric artery segments before (A) and after (B) incubation with the nitric oxide synthase inhibitor $\mathrm{N}^{\mathrm{G}}$-nitro-L-arginine methyl ester (L-NAME) and the cyclooxygenase inhibitor indomethacin (INDO). Mean values of the $\mathrm{pD}_{2}$ of $\mathrm{ACh}$ in the absence of L-NAME and INDO were $7.56 \pm 0.08$ (Control), $7.42 \pm 0.06$ (Stress) and in the presence of L-NAME and INDO were $6.27 \pm 0.03$ (Control+L-NAME+INDO, $p<0.01 v s$. Control) and $6.58 \pm 0.03$ (Stress+L-NAME+INDO, $p<0.01 v s$. Stress). Values represent mean \pm SEM of 5-6 rats. Data were analysed by two-way ANOVA test, followed by Bonferroni post hoc test. ${ }^{+} p<0.05,{ }^{++} p<0.01$, compared to respective value in control or stressed rats without L-NAME + INDO. $\mathrm{pD}_{2}$, negative logarithm of the molar concentration of agonist producing 50 percent of maximal response.

tions induced by endogenous noradrenaline on SMA rings is illustrated in Fig. 4B,C. Stress significantly elevated neurogenic contractions compared to the control group (Fig. 4B), however, contractions were similar when calculated to maximal contractions induced by depolarising solution (Fig. 4C). Additionally, contractions to depolarising solution were significantly greater from stressed than from control rats (data not shown).

\section{Endothelium-dependent relaxation of the SMA}

There were no differences in the responses of SMA to acetylcholine before or after pre-incubation with L-NAME and indomethacin between the control and stressed group (Fig. $5 \mathrm{~A}, \mathrm{~B})$. Blockade of $\mathrm{NO}$ synthesis by L-NAME and $\mathrm{PGI}_{2}$ synthesis by indomethacin reduced significantly relaxations to acetylcholine (Fig. 5B). Crowding had no significant effect on endothelium-independent sodium nitroprussideinduced relaxation of the SMA (data not shown).

\section{Discussion}

The study investigated the effect of chronic social stress produced by crowding on mesenteric arteries and its role in the initiation of stress-related hypertension. The obtained data showed that the given paradigm of chronic crowding reduced body weight gain and plasma triglycerides but failed to affect blood pressure, heart rate, relative left ventricular weight, diameter of 1MA, resting 1MA wall tension and cholesterol content in the liver, skeletal muscle and plasma in normotensive rats. Additionally, investigation of mesenteric arterial function showed only a mild effect of crowding in this vasculature. Increased constrictor responses induced by exogenous and endogenous noradrenaline were found in SMA. However, neither endothelium-dependent vasorelaxation of SMA nor maximal vasorelaxation and constriction of the first branches of the mesenteric artery were affected by stress in normotensive rats.

This study is in agreement with previous results which showed unaltered blood pressure during chronic crowding in normotensive Wistar rats. Yet the same model of stress induced hypertension in offspring of spontaneously hypertensive (SHR) mothers mated with Wistar males while offspring of normotensive Wistar mothers mated with SHR males were able to adapt to stress (Bernatova et al. 2007a). Similarly to our findings, other authors failed to observe changes in blood pressure during chronic psychosocial stress in normotensive rats (Harrap et al. 1984; Henry et al. 1993), 
while some experimental models of social stress were able to induce hypertension in normotensive strains (Webb et al. 1987; Henry et al. 1993; Andrews et al. 2003).

The endothelium plays an important role in cardiovascular homeostasis. A potential negative influence of stress on the endothelium was observed in both humans (Sherwood et al. 1999; Ghiadoni et al. 2000) and animals (Strawn et al. 1991; Williams et al. 1993; Jezova et al. 2003; Okruhlicová et al. 2008; Neves et al. 2009). Findings of Wallerath et al. (1999) and Rogers et al. (2002) suggest involvement of stress-induced glucocorticoid secretion in regulation of vascular NO synthesis. The former authors observed reduced expression of endothelial NOS in the aorta of rats chronically treated with dexamethasone (a substitute for endogenous glucocorticoid). In this context, it was also shown that glucocorticoids inhibited the expression of inducible, but not the constitutive NOS in vascular endothelial cells without direct effect on the activity of either of them (Radomski et al. 1990). In contrast, we observed elevated aortic NOS activity and improved endothelium-dependent relaxation, including its NO-dependent component, in the femoral artery of normotensive rats exposed to crowding (Púzserová et al. 2006; Bernátová et al. 2007b). Improved NO-dependent relaxation in crowded rats was observed in the femoral artery both in the presence and absence of AsA in working PSS, suggesting no significant influence of oxidative stress on elevated NO level in this artery (Puzserova and Bernatova 2010). Interestingly, the improvement of acetylcholine-induced relaxation in 1MA was seen only in the presence of AsA (Bernátová et al. 2007b), while in this study, where AsA was removed, no considerable effect of stress was observed. This suggests that AsA may modify vascular reactivity in 1MA. In the absence of AsA, reactive oxygen species may presumably block the improvement of NO-dependent relaxation induced by stress in the mesenteric bed. Since chronic stress can lead to increasing generation of reactive oxygen species (Kwiecien et al. 2008), antioxidants, including AsA, might artificially modify endothelial function by improving the oxidative status and NO bioavailability in vivo (May 2000).

Concerning endothelial function, there are studies showing that psychosocial stress was associated with endothelial dysfunction in primates (Strawn et al. 1991). In humans, a brief episode of mental stress caused transient endothelial dysfunction in healthy young individuals (Ghiadoni et al. 2000). In cynomolgus monkeys, chronic social stress resulted in a reduced NO-dependent relaxation of atherosclerotic iliac arteries (Williams et al. 1993). On the other hand, increased vasodilator responses to acetylcholine of the methoxamine-contracted aorta were observed in mice (Webb et al. 1987) and an unchanged response was found in the small mesenteric arteries of normotensive rats (Fuchs et al. 1998) exposed to chronic social and behavioural stress. Recently, Neves et al. (2009) reported that chronic mild unpredictable stress induced simultaneously functional and morphological changes in the Sprague-Dawley rat thoracic aorta, suggesting that these functional effects may be related to decreased synthesis of NO. On the contrary, our experiments (Bernátová et al. 2007b) showed that chronic crowding increased NO synthase activity and nitrate/nitrite levels in the aorta.

However, similarly to NO, stress may also affect the release of other endothelium-derived relaxing factors. Fuchs et al. (1998) observed that stress enhanced the role of vasodilatory cyclooxygenase products in the rat small mesenteric arteries. Yet in our study, acetylcholine-induced relaxations of 1MA from crowded rats were unaltered after pre-incubation of the arteries with L-NAME, indicating that the endothelium produced similar amounts of L-NAME-resistant relaxing factors, such as prostacycline and/or endothelium-derived hyperpolarising factors (Mitchell et al. 2008). Similarly, no functional changes of the endothelium in the SMA were seen after crowding. Thus the results suggest that chronic crowding stress exposure did not modify bioavailability of endothelial factors in either 1MA or SMA. On the other hand, Chies et al. (2003) reported that forced swimming stress may increase non-endothelial NO activity in both the aorta and SMA of adult male Wistar rats. In the light of other findings, the vascular adaptive response to stress was characterised by hyperactivity of the endothelial L-arginine/NO system in male Wistar rats (Cordellini and Vassilieff 1998; Cordellini et al. 2006). Another study showed increased response to acetylcholine in the aorta after acute immobilisation (Cordellini and Vassilieff 1998). Additionally, Webb et al. (1987) found that the mean depressor responses to acetylcholine in the hindquarters of psychosocially hypertensive CBA Agouti male mice were significantly greater than those in normotensive mice. However, depressor responses to the NO donor sodium nitroprusside in stressed mice were not significantly different from control mice (Webb et al. 1987), which is in agreement with our findings of unaltered endothelium-independent relaxation to $\mathrm{NO}$ donor in stressed rats.

Regarding vasoconstriction, we examined responses to phenylephrine, noradrenaline and neurogenic contractions induced by perivascular nerve stimulation. Our results showed no significant changes regarding maximal noradrenaline and phenylephrine vasoconstriction of 1MA in stressed rats compared to controls. Similar results were also obtained in male WKY rats, in which 10-day exposure to behavioural stress resulted in unchanged responsiveness to the $\alpha_{1}$-adrenoceptor agonist phenylephrine in small mesenteric arteries (Fuchs et al. 1998). However, it was also observed that pre-incubation of 1MA with L-NAME induced no significant enhancement of the vasoconstrictor potency of phenylephrine, indicating smaller functionality of NO in this type of artery. In the present study, stress significantly elevated vasoconstriction to exogenous noradrenaline and increased 
neurogenic contractions induced by endogenous noradrenaline in SMA, in spite of AsA presence in PSS. Several drugs have to be dissolved in AsA solution to prevent degradation of unstable substances, for example catecholamines (Bernatova et al. 2009; Neves et al. 2009). This is the reason, why we are used the modified physiological solution with AsA for exogenous noradrenaline and neurogenic contraction analysis in an organ chamber experiment. In accordance with findings of Neves et al. (2009), we suggest that since endothelium-dependent relaxation was not changed, structural changes and/or increased sensitivity to noradrenaline may account for augmented contractile responses to exogenous and endogenous noradrenaline in SMA.

Hypercholesterolemia was assumed to represent a link between stress, endothelial dysfunction and atherogenesis (Neves et al. 2009). In this study, stress failed to affect plasma levels of TC, LDL and HDL in rats, which corresponded well with unchanged liver and skeletal muscle cholesterol content. Unaltered AI (an index evaluating the risk of atherosclerosis (Neves et al. 2009)) in stressed rats was in accordance with intact endothelial function in both arteries investigated. Intriguingly, however, TGL and VLDL levels were reduced in stressed animals. This may result from decreased body weight gain, due to decreased food intake (Púzserová et al. 2008).

Discrepancies in the effect of chronic stress on blood pressure and endothelial function appearing in different studies may reflect the use of various stressors and animal models. Moreover, the data suggest that reaction to stressors could be specific, depending on the nature, duration and intensity of the stressor, animal strain, age, gender, etc. (Djordjević et al. 2003). On balance, the exact mechanism(s) responsible for the development of stress-induced cardiovascular disorders remain still unknown.

In conclusion, chronic stress produced by crowding increased neurogenic and exogenous noradrenaline-induced contractions in SMA of adult normotensive rats. Nevertheless, stress failed to induce an increase of blood pressure, presumably because endothelial function of SMA and vascular function of small mesenteric arteries, which play an even more important role in blood pressure regulation, remained preserved.

Acknowledgement. The authors thank to Peter Slezak, MSc. for his statistical advice and to Mrs. Jana Petova for her technical assistance. This study was supported by the Slovak Grant Agency for Science, grants No. 2/0084/10, No. 2/0193/10 and No. 2/0086/08 and APVV-0523-10.

\section{References}

Andrews E., Jenkins C., Seachrist D., Dunphy G., Ely D. (2003) Social stress increases blood pressure and cardiovascular pa- thology in a normotensive rat model. Clin. Exp. Hypertens. 25, 85-101

http://dx.doi.org/10.1081/CEH-120017930

Bernatova I., Csizmadiova Z., Kopincova J., Puzserova A. (2007a): Vascular function and nitric oxide production in chronic socialstress-exposed rats with various family history of hypertension. J. Physiol. Pharmacol. 58, 487-501

Bernátová I., Púzserová A., Navarová J., Csizmadiová Z., Zeman M. (2007b): Crowding-induced alterations in vascular system of Wistar-Kyoto rats: role of nitric oxide. Physiol. Res. 56, 667-669

Bernatova I., Conde M. V., Kopincova J., González M. C., Puzserova A., Arribas S. M. (2009): Endothelial dysfunction in spontaneously hypertensive rats: focus on methodological aspects. J. Hypertens. 27, S27-31 http://dx.doi.org/10.1097/01.hjh.0000358834.18311.fc

Bernatova I., Puzserova A., Dubovicky M. (2010): Sex differences in social stress-induced pressor and behavioral responses in normotensive and prehypertensive rats. Gen. Physiol. Biophys. 29, 346-354 http://dx.doi.org/10.4149/gpb_2010_04_346

Bugajski A.J., Gadek-Michalska A., Bugajski J. (2006): The involvement of nitric oxide and prostaglandins in the cholinergic stimulation of hypothalamic-pituitary-adrenal response during crowding stress. J. Physiol. Pharmacol. 57, 463-477

Bugajski J. (1999): Social stress adapts signaling pathways involved in stimulation of the hypothalamic-pituitary-adrenal axis. J. Physiol. Pharmacol. 50, 367-379

Čačányiová S., Pecháňová O., Babál P., Černá A., Janega P., Andriantsitohaina R. (2011): Red wine polyphenols correct vascular function injured by chronic carbon tetrachloride intoxication. Gen. Physiol. Biophys. 30, 207-213

http://dx.doi.org/10.4149/gpb_2011_02_207

Caramori P. R., Zago A. J. (2000): Endothelial dysfunction and coronary artery disease. Arq. Bras. Cardiol. 75, 163-182 http://dx.doi.org/10.1590/S0066-782X2000000800009

Chies A. B., Corrêa F. M., de Andrade C. R., Rosa-e-Silva A. A., Pereira F. C., de Oliveira A. M. (2003): Vascular non-endothelial nitric oxide induced by swimming exercise stress in rats. Clin. Exp. Pharmacol. Physiol. 30, 951-957 http://dx.doi.org/10.1111/j.1440-1681.2003.03935.x

Cordellini S., Vassilieff V. S. (1998): Decreased endothelium-dependent vasoconstriction to noradrenaline in acute-stressed rats is potentiated by previous chronic stress: nitric oxide involvement. Gen. Pharmacol. 30, 79-83 http://dx.doi.org/10.1016/S0306-3623(97)00074-8

Cordellini S., Novo R., Lanza U., Jr. (2006): Exposure to stress differential vascular adaptive response in spontaneously hypertensive and Wistar rats: Role of nitric oxide, and prehypertensive and hypertensive states. Life Sci. 79, 646-653 http://dx.doi.org/10.1016/j.lfs.2006.02.007

D’Atri D. A., Fitzgerald E. F., Kasl S. V., Ostfeld A. M. (1981): Crowding in prison: the relationship between changes in housing mode and blood pressure. Psychosom. Med. 43, 95-105

Djordjević J., Cvijić G., Davidović V. (2003): Different activation of ACTH and corticosterone release in response to various stressors in rats. Physiol. Res. 52, 67-72

Dronjak S., Gavrilović L., Filipović D., Radojcić M. B. (2004): Immobilization and cold stress affect sympatho-adrenomedul- 
lary system and pituitary-adrenocortical axis of rats exposed to long-term isolation and crowding. Physiol. Behav. 81, 409-415

http://dx.doi.org/10.1016/j.physbeh.2004.01.011

Esch T., Stefano G. B., Fricchione G. L., Benson H. (2002): Stressrelated diseases - a potential role for nitric oxide. Med. Sci. Monit. 8, RA103-118

Friedewald W. T., Levy R. I., Fredrickson D. S. (1972): Estimation of the concentration of low-density lipoprotein cholesterol in plasma, without use of the preparative ultracentrifuge. Clin. Chem. 18, 499-502

Fuchs L. C., Hoque A. M., Clarke N. L. (1998): Vascular and hemodynamic effects of behavioral stress in borderline hypertensive and Wistar-Kyoto rats. Am. J. Physiol. 274, R375-382

Gadek-Michalska A., Bugajski J. (2008): Nitric oxide in the adrenergic- and CRH-induced activation of hypothalamic-pituitaryadrenal axis. J. Physiol. Pharmacol. 59, 365-378

Gadek-Michalska A., Bugajski A. J., Bugajski J. (2008): Nitric oxide and prostaglandins in the clenbuterol-induced ACTH and corticosterone secretion. J. Physiol. Pharmacol. 59, $163-175$

Gerová M., Masánová C., Pavlásek J. (1995): Inhibition of NO synthase in the posterior hypothalamus increases blood pressure in the rat. Physiol. Res. 44, 131-134

Ghiadoni L., Donald A. E., Cropley M., Mullen M. J., Oakley G., Taylor M., O’Connor G., Betteridge J., Klein N., Steptoe A., Deanfield J. E. (2000): Mental stress induces transient endothelial dysfunction in humans. Circulation 102, 2473-2478 http://dx.doi.org/10.1161/01.CIR.102.20.2473

Hansen K., Nedergaard O. A. (1999): Methodologic aspects of acetylcholine-evoked relaxation of rabbit aorta. J. Pharmacol. Toxicol. Methods 41, 153-159 http://dx.doi.org/10.1016/S1056-8719(99)00035-0

Harrap S. B., Louis W. J., Doyle A. E. (1984): Failure of psychosocial stress to induce chronic hypertension in the rat. J. Hypertens. 2, 653-662 http://dx.doi.org/10.1097/00004872-198412000-00011

Henry J. P., Liu Y. Y., Nadra W. E., Qian C. G., Mormede P., Lemaire V., Ely D., Hendley E. D. (1993): Psychosocial stress can induce chronic hypertension in normotensive strains of rats. Hypertension 21, 714-723 http://dx.doi.org/10.1161/01.HYP.21.5.714

Jezova D., Kristova V., Slamova J., Mlynarik M., Pirnik Z., Kiss A., Kriska M. (2003): Stress-induced rise in endothelaemia, von Willebrand factor and hypothalamic-pituitary-adrenocortical axis activation is reduced by pretreatment with pentoxifylline. J. Physiol. Pharmacol. 54, 329-338

Kamgang R., Mboumi R. Y., N’dillé G. P., Yonkeu J. N. (2005): Cameroon local diet induced glucose intolerance and dyslipidemia in adult Wistar rat. Diabetes Res. Clin. Pract. 69, 224-230 http://dx.doi.org/10.1016/j.diabres.2005.02.005

Kaplan J. R., Manuck S. B., Clarkson T. B., Lusso F. M., Taub D. M., Miller E. W. (1983): Social stress and atherosclerosis in normocholesterolemic monkeys. Science 220, 733-735 http://dx.doi.org/10.1126/science.6836311

Kwiecien S., Pawlik M. W., Brzozowski T., Konturek P. C., Sliwowski Z., Pawlik W. W., Konturek S. J. (2008): Nitric oxide (NO)-releasing aspirin and other NO donors in protection of gastric mucosa against stress. J. Physiol. Pharmacol. 59, 103-115

Leza J. C., Salas E., Sawicki G., Russell J. C., Radomski M. W. (1998): The effects of stress on homeostasis in JCR-LA-cp rats: the role of nitric oxide. J. Pharmacol. Exp. Ther. 286, 1397-1403

Malyshev I. Y., Manukhina E. B. (1998): Stress, adaptation, and nitric oxide. Biochemistry (Mosc.) 63, 840-853

May J. M. (2000): How does ascorbic acid prevent endothelial dysfunction? Free. Radic. Biol. Med. 28, 1421-1429 http://dx.doi.org/10.1016/S0891-5849(00)00269-0

Mitchell J. A., Ali F., Bailey L., Moreno L., Harrington L. S. (2008): Role of nitric oxide and prostacyclin as vasoactive hormones released by the endothelium. Exp. Physiol. 93, 141-147 http://dx.doi.org/10.1113/expphysiol.2007.038588

Mulvany M. J., Halpern W. (1977): Contractile properties of small arterial resistance vessels in spontaneously hypertensive and normotensive rats. Circ. Res. 41, 19-26 http://dx.doi.org/10.1161/01.RES.41.1.19

Neves V. J., Moura M. J., Tamascia M. L., Ferreira R., Silva N. S., Costa R., Montemor P. L., Narvaes E. A., Bernardes C. F., Novaes P. D., Marcondes F. K. (2009): Proatherosclerotic effects of chronic stress in male rats: altered phenylephrine sensitivity and nitric oxide synthase activity of aorta and circulating lipids. Stress 12, 320-327 http://dx.doi.org/10.1080/10253890802437779

Okruhlicová L., Dlugosová K., Mitasíková M., Bernátová I. (2008): Ultrastructural characteristics of aortic endothelial cells in borderline hypertensive rats exposed to chronic social stress. Physiol. Res. 57, S31-37

Puzserova A., Bernatova I. (2010): Chronic social stress increases nitric oxide-dependent vasorelaxation in normotensive rats. Interdiscip. Toxicol. 3, 109-117 http://dx.doi.org/10.2478/v10102-010-0049-4

Púzserová A., Csizmadiová Z., Andriantsitohaina R., Bernátová I. (2006): Vascular effects of red wine polyphenols in chronic stress-exposed Wistar-Kyoto rats. Physiol. Res. 55, S39-47

Púzserová A., Kopincová J., Bernátová I. (2008): Social stress impact on blood pressure, vascular function, food and water intake in Wistar-Kyoto rats. In: Topics in Higher Brain Functions. (Eds. F. Jagla and I. Riečanský), pp. 122-126, Comenius University, Bratislava

Radomski M. W., Palmer R. M., Moncada S. (1990): Glucocorticoids inhibit the expression of an inducible, but not the constitutive, nitric oxide synthase in vascular endothelial cells. Proc. Natl. Acad. Sci. U.S.A. 87, 10043-10047 http://dx.doi.org/10.1073/pnas.87.24.10043

Rogers K. M., Bonar C. A., Estrella J. L., Yang S. (2002): Inhibitory effect of glucocorticoid on coronary artery endothelial function. Am. J. Physiol. Heart Circ. Physiol. 283, H1922-1928

Scheuer D. A. (2010): Adrenal corticosteroid effects in the central nervous system on long-term control of blood pressure. Exp. Physiol. 95, 10-12 http://dx.doi.org/10.1113/expphysiol.2008.045484

Sherwood A., Johnson K., Blumenthal J. A., Hinderliter A. L. (1999): Endothelial function and hemodynamic responses during mental stress. Psychosom. Med. 61, 365-370

Siddegowda Y. K., Leo M. D., Kumar D., Hooda O. K., Prakash V. R., Mishra S. K. (2007): Influence of heat stress on the reactiv- 
ity of isolated chicken carotid artery to vasoactive agents. Exp. Physiol. 92, 1077-1086 http://dx.doi.org/10.1113/expphysiol.2007.038844

Sotnikova R., Skalska S., Okruhlicova L., Navarova J., Kyselova Z., Zurova J., Stefek M., Hozova R., Nosalova V. (2006): Changes in the function and ultrastructure of vessels in the rat model of multiple low dose streptozotocin-induced diabetes. Gen. Physiol. Biophys. 25, 289-302

Strawn W. B., Bondjers G., Kaplan J. R., Manuck S. B., Schwenke D. C., Hansson G. K., Shively C. A., Clarkson T. B. (1991): Endothelial dysfunction in response to psychosocial stress in monkeys. Circ. Res. 68, 1270-1279 http://dx.doi.org/10.1161/01.RES.68.5.1270

Török J. (2008): Participation of nitric oxide in different models of experimental hypertension. Physiol. Res. 57, 813-825

Ulicná O., Greksák M., Vancová O., Zlatos L., Galbavý S., Bozek P., Nakano M. (2003): Hepatoprotective effect of rooibos tea (Aspalathus linearis) on CCl4-induced liver damage in rats. Physiol. Res. 52, 461-466
Wallerath T., Witte K., Schäfer S. C., Schwarz P. M., Prellwitz W., Wohlfart P., Kleinert H., Lehr H. A., Lemmer B., Förstermann U. (1999): Down-regulation of the expression of endothelial NO synthase is likely to contribute to glucocorticoid-mediated hypertension. Proc. Natl. Acad. Sci. U.S.A. 96, 13357-13362 http://dx.doi.org/10.1073/pnas.96.23.13357

Webb R. C., Vander A. J., Henry J. P. (1987): Increased vasodilator responses to acetylcholine in psychosocial hypertensive mice. Hypertension 9, 268-276 http://dx.doi.org/10.1161/01.HYP.9.3.268

Williams J. K., Kaplan J. R., Manuck S. B. (1993): Effects of psychosocial stress on endothelium-mediated dilation of atherosclerotic arteries in cynomolgus monkeys. J. Clin. Invest. 92, 1819-1823

http://dx.doi.org/10.1172/JCI116772

Received: June 29, 2011

Final version accepted: April 3, 2012 